\title{
Central Auditory Processing Deficits in the Elderly
}

\author{
Lilian Felipe* \\ Speech and Hearing Department, Lamar University, USA \\ *Corresponding author: Lilian Felipe, Speech and Hearing Department, Lamar University, USA \\ To Cite This Article: Lilian Felipe. Central Auditory Processing Deficits in the Elderly. Am J Biomed Sci \& Res. 2019 - 3(2). AJBSR.MS.ID.000654. \\ DOI: 10.34297/AJBSR.2019.03.000654
}

Received: March 06, 2019 | Published: May 28, 2019

\begin{abstract}
Elderly persons with and without hearing loss experience difficulty listening to speech in background noise, following rapid speech, attending to multiple speakers, or understanding complex messages. When the listening situation is complex or challenging the person expands additional listening effort, which can lead to fatigue, reduced attention, and forgetfulness. Beyond the age-related effects on hearing, known as presbycusis, the central auditory system suffers decline in processing abilities with memory and attention. This common occurrence for the aging brain to experience some decline in functioning, is known as, "central presbycusis". Specifically related to the auditory system, the brain declines in its ability to listen to speech in noise, dichotic processing which is attending to stimuli in one/both/alternating ears, and temporal processing which is attending to rapid changes in stimuli. Research shows that engaging in cognitively demanding activities like reading, learning, playing games, playing chess, speaking another language, being physically active, engaging in social activities, and playing music can delay and/or reduce cognitive decline and dementia. This paper will further elaborate on the effects of central auditory processing deficits resulting from central presbycusis in the elderly.
\end{abstract}

Keywords: Aging; Central auditory processing deficits; Central presbycusis; Cognitive decline; Dichotic listening; Elderly; Temporal processing

\section{Introduction}

Age related effects on the peripheral auditory system is commonly discussed in relation to presbycusis, but a central presbycusis can also occur [1]. Central presbycusis is often suspected when an elderly person displaces disproportionally poor speech performance when considering their hearing sensitivity, physical health, and cognitive status. The central auditory nervous system (CANS) comprises of the anatomical and physiological processes between the cochlear nuclei in the brainstem, up through the auditory cortex located in the temporal lobe of the brain where the primary auditory processing centers, Heschl's gyrus and Sylvian fissure are located [2]. The CANS is responsible for identifying incoming signal, stimuli localization, auditory discrimination, masking, integration, temporal processing, ordering, and attending to desired stimuli in competing acoustic signals or degraded acoustic signal [3].

Correspondingly, when considering the processing of speech comprehension, one must separate the effect of the peripheral system to supply appropriate "bottom-up" data for processing, and the central auditory system to perform "top-down" higher-order process $[1,4]$. The neural strength needed to process a puretone is far less than that required to comprehend speech, especially in a complex or degraded listening condition. In fact, results of the audiogram very limitedly expose the functioning ability or inability of the persons complete auditory system [2]. However, it is known that cochlear degeneration can negatively impact resolution of spectral and temporal processing of an auditory signal [5]. General cognitive functions that can further impact comprehension are memory, attention, and speech of processing [1].

Symptoms commonly reported with auditory processing deficits include: difficulty understanding speech in noise, difficulty on the telephone, difficulty following conversations (especially with multiple talkers), difficulty following rapid speech, hearing someone is talking but do not understand what is being said, and lack of music appreciation [2,4]. Decline in production and comprehension of complex messages can be attributed to the agerelated decline of working memory [4]. Furthermore, increased listening effort required to attend to complex or degraded signals can reallocate cognitive load resulting in poor short-term and longterm memory [4,5]. Prevalence of auditory processing deficits vary considerably depending on severity, other comorbid health conditions, effect of hearing sensitivity, cognitive impairments, etc. [6].

\section{Discussion}

While the symptoms and effects of a central auditory processing deficit can be broad, specific psychophysical tests can have been useful in evaluating the deficits. Primarily, speech-in-noise testing, dichotic listening, and temporal processing assessments can be 
used. Speech-in-noise testing is a very common complaint of the elderly. It has been found that the decrease in ability does not proceed in a linear fashion, but instead accelerates with advancing age [1]. Use of a speech-in-noise test in the clinical setting may provide the clinician with a more accurate representation of how the individual is performing in daily living conditions.

Next, dichotic listening is a primary area of difficulty when considering the need to attend to specific signals in either ear, which is especially important for understanding speech in noise settings. Dichotic Sentence Identification tests with both divided-attention and directed-attention can be used for evaluation. Assessment of dichotic listening found that some elderly persons have a left-ear disadvantage and poor message-to-comprehension scores as the listening environment became more challenging which supports the possibility of declined functioning of the corpus callosum [4].

Ability to use and maintain attention for dichotic listening becomes increasingly important with age [4].

Also, the ability to perform accurate and timely temporal processing of the minute differences in stimuli aids in understanding the difference between phonemes, speech in complex listening situations, rapid speech, and degraded speech signals [4]. It has been found that even a small degree of peripheral hearing loss can impact gap detection abilities, but also decline in gap detection abilities can occur independent of peripheral hearing loss [1].

A comprehensive audiologic evaluation is recommended, but additional electrophysiologic tests can be useful in assessing the elderly population. The auditory brainstem response (ABR) may show normal latencies with reduced amplitudes [1]. Middle latency response (MLR) test may show increased amplitudes due to the greater decrease in inhibitory rather than excitatory neurons [1]. Also, late latency responses (LLR) may require longer gaps with the mismatch negativity (MMN) in order to elicit an MMN response. The MMN response and P2 wave may also show reduced amplitude and delayed latencies; but the results of the P300 wave are variable $[1,7]$.

Rehabilitation and treatment can include amplification, environmental modifications, communication strategy and compensatory strategy training, education and counseling, and self-advocacy $[1,2]$. Any effect to improve the listening condition will subsequently reduce cognitive load, which will reduce listening effort and free cognitive resources for memory [6]. Comprehension abilities are limited by the ability of the person to make use of gaps in noise and vary depending on the type of task and complexity of the information being delivered [2]. Older adults that engaging in cognitively demanding activities like reading, learning, playing games, playing chess, speaking another language, being physically active, engaging in social activities, and playing music can delay and/or reduce cognitive decline and dementia [6].

\section{Conclusion}

Central auditory deficits are a natural occurrence with aging with common overlapping complaints. However, effect of this decline, known as central presbycusis is highly variable. These deficits can occur with and without the presence of hearing loss. Any hearing loss present will surely exacerbate the central auditory important. Additionally, and cognitive declines will hinder communicative abilities and understanding. Any effort to improve the auditory input with environmental modifications, communication strategies or amplification will improve the "bottom-up" processing of the signal. This will reduce cognitive load and support the "top-down" processing of the information and hopefully improve memory function.

\section{References}

1. Atcherson SR, Nagarag NK, Kennet SEW, Levisee M (2015) Overview of central auditory processing deficits in older adults. Semin Hear 36(3): 150-161.

2. Larsby B, Hällgren M, Lyxell B, Whitelaw (2008) The interference of different background noises on speech processing in elderly hearingimpaired subjects. Int J Audiol 47(Suppl 2): S83-S90.

3. American Speech-Language Hearing Association (ASHA) Task Force on Central Auditory Processing Consensus Development. (1996) Central auditory processing: Current status of research and implications for clinical practice. American Journal of Audiology 5(2): 41-54.

4. Martin JS, Jerger JF (2005) Some effects of aging on central auditory processing. J Rehabil Res Dev 42(4 Suppl 2): 25-43.

5. Murphy DR, Schneider BA, Speranza F, Moraglia G (2006) A Comparison of Higher Order Auditory Processes in Younger and Older Adults. Psychol Aging 21(4): 763-773.

6. Zendel RB, Alain C (2012) Musicians Experience Less Age-Related Decline in Central Auditory Processing. Psychol Aging 27(2): 410-417.

7. Stenklev NC, Laukli E (2004) Cortical Cognitive Potentials in Elderly Persons. J Am Acad Audiol 15(6): 401-413. 